\title{
Vessel Wall Magnetic Resonance Imaging Findings of Bilateral Middle Cerebral Artery Stenosis Associated with Nilotinib
}

\author{
Sungjoon Yoon, MD (D); Seon Deuk Kim, MD (D; Juhee Lee, MD (D); Mu Seong Kim, MD (D); \\ Yong Woo Shin, MD (D; Eung-Joon Lee, MD (D) \\ Department of Neurology, Seoul National University Hospital, Seoul National University College of Medicine, Seoul, Korea
}

Nilotinib is a Bcr-Abl tyrosine kinase inhibitor used to treat chronic myelogenous leukemia. There have been case reports of nilotinib-related vasculopathy. However, most cases present peripheral artery disease, whilst reports of nilotinib-related cerebrovascular disease are quite uncommon. Herein, we report a case of nilotinib-induced intracranial stenosis in a patient with recurrent transient ischemic attacks and discuss the results of serial vessel wall magnetic resonance imaging.

J Neurosonol Neuroimag 2021;13(2):So-83

Key Words: Transient ischemic attack; transient; Protein kinase inhibitors; Nilotinib; Cerebrovascular disorders; Atherosclerosis

Nilotinib is a Bcr-Abl tyrosine kinase inhibitor (TKI) used to treat chronic myelogenous leukemia (CML). There have been case reports of nilotinib-related vasculopathy. ${ }^{1}$ However, a report of nilotinib-related cerebrovascular disease (CVD) is somewhat unusual. ${ }^{2-4}$ There are no previous reports of a case with serial vessel wall magnetic resonance imaging (VWI). Herein, we report a case of nilotinib-induced intracranial stenosis in a patient with recurrent transient ischemic attacks (TIAs) and discuss the results of VWI.

\section{CASE}

A 46-year-old man visited the emergency department because of four recurrent episodes of transient left-sided weakness since 1 day prior. Each event persisted for a few minutes. He was diagnosed with CML 13 years prior, and imatinib therapy was started at that time. A positive conversion of the Bcl-abl transcript prompted a switch to $400 \mathrm{mg}$ nilotinib twice a day. The patient maintained nilotinib for 8 years, and the chronic phase
Received: November 10, 2021 Revised: December 9, 2021 Accepted: December 13, 2021

Correspondence:

Eung-Joon Lee, MD

Department of Neurology, Seoul National University Hospital, Seoul National University College of Medicine, 101 Daehak-ro, Jongno-gu, Seoul 03080, Korea

Tel: $+82-2-2072-2278$

Fax: +82-2-2072-1106

E-mail: lejoono824@gmail.

com of CML was well controlled. The man had never been a smoker and had no significant risk factors for CVD; therefore, it was assumed that his 10-year atherosclerotic cardiovascular disease risk was lower than $1.3 \%$. In addition, he had never experienced symptoms suggestive of peripheral artery disease. Neurological examination revealed no focal neurological deficits. Brain magnetic resonance imaging and angiography revealed no focal parenchymal abnormalities; however, significant bilateral stenosis of the middle cerebral arteries (MCAs) was noted (Fig. 1A). In this context, the patient was diagnosed with transient ischemic attacks (TIAs), and significant stenosis of the right MCA was considered the culprit lesion with a clinical correlation. A diagnostic workup for bilateral MCA stenosis was performed. His low-density lipoprotein cholesterol level was $97 \mathrm{mg} / \mathrm{dL}$, and his glycated hemoglobin level was 5.9\%. Blood test results for vasculitis, including antinuclear antibodies, antineutrophil cytoplasmic antibodies, antiphospholipid antibodies, antithrombin III, and protein C/S were all negative. VWI targeting the bilateral MCA was performed to differentiate the etiology (Ingenia CX 3.0 T, 
Philips Medical Systems, Netherlands). Eccentric wall thickening of the right MCA with high signal intensity was revealed via proton density imaging (PDI). The following values list the scanning parameters for PDI: $T R=1,800.0 \mathrm{~ms} ; \mathrm{TE}=36.1 \mathrm{~ms}$ and $\mathrm{T} 1$ contrast enhancement: TR=650.0 ms; TE=37.1 ms, respectively (Fig. 1B1D). Since VWI suggested intracranial atherosclerosis, other pathologies, including dissection and Moyamoya disease, were excluded.

For the secondary prevention of ischemic stroke, a daily dose of $75 \mathrm{mg}$ clopidogrel was prescribed, and the regimen of $400 \mathrm{mg}$ nilotinib twice daily was switched to a daily dose of $100 \mathrm{mg}$ dasatinib. The Bcl-abl transcript was detected every three months. A follow-up test 21 months after replacing nilotinib with dasatinib showed a positive conversion of the Bcl-abl transcript. Furthermore, the patient did not experience any neurological symptoms. A VWI follow-up 2 years after the TIA episodes showed no progression of MCA stenosis with slightly decreased wall enhancement and a high signal PDI compared to the first imaging result (Ingenia CX 3.0T, identical protocol) (Fig. $1 \mathrm{E}-\mathrm{G}$ ).

\section{DISCUSSION}

Nilotinib, a front-line therapy for chronic CML, mainly targets the BCR-ABL gene. Systemic vasculopathy is a known adverse effect of TKIs. Although peripheral artery diseases are most commonly reported, ${ }^{1}$ cerebrovascular events associated with nilotinib have also been described. ${ }^{2-4}$ However, the mechanisms by which nilotinib promotes atherosclerosis remain poorly understood. According to previous studies, nilotinib induces pro-atherogenic and anti-angiogenic effects on endothelial cells by binding to discoidin domain receptor 1, platelet-derived growth factor receptor, and C-kit. ${ }^{1}$ Nilotinib exerts pro-inflammatory effects by upregulating pro-inflammatory cytokines ${ }^{5}$ and worsening glycemic control ${ }^{6}$ and lipid profiles. ${ }^{7}$ Thus, nilotinib may accelerate systemic arterial stenosis via an atherosclerotic etiology. It also has pro-thrombogenic effects by increasing platelet adhesion and downregulating mast cells, which secrete tissue plasminogen activator and heparin, ${ }^{8}$ which may also contribute to clinical vascular events. The results of VWI in our case suggest-
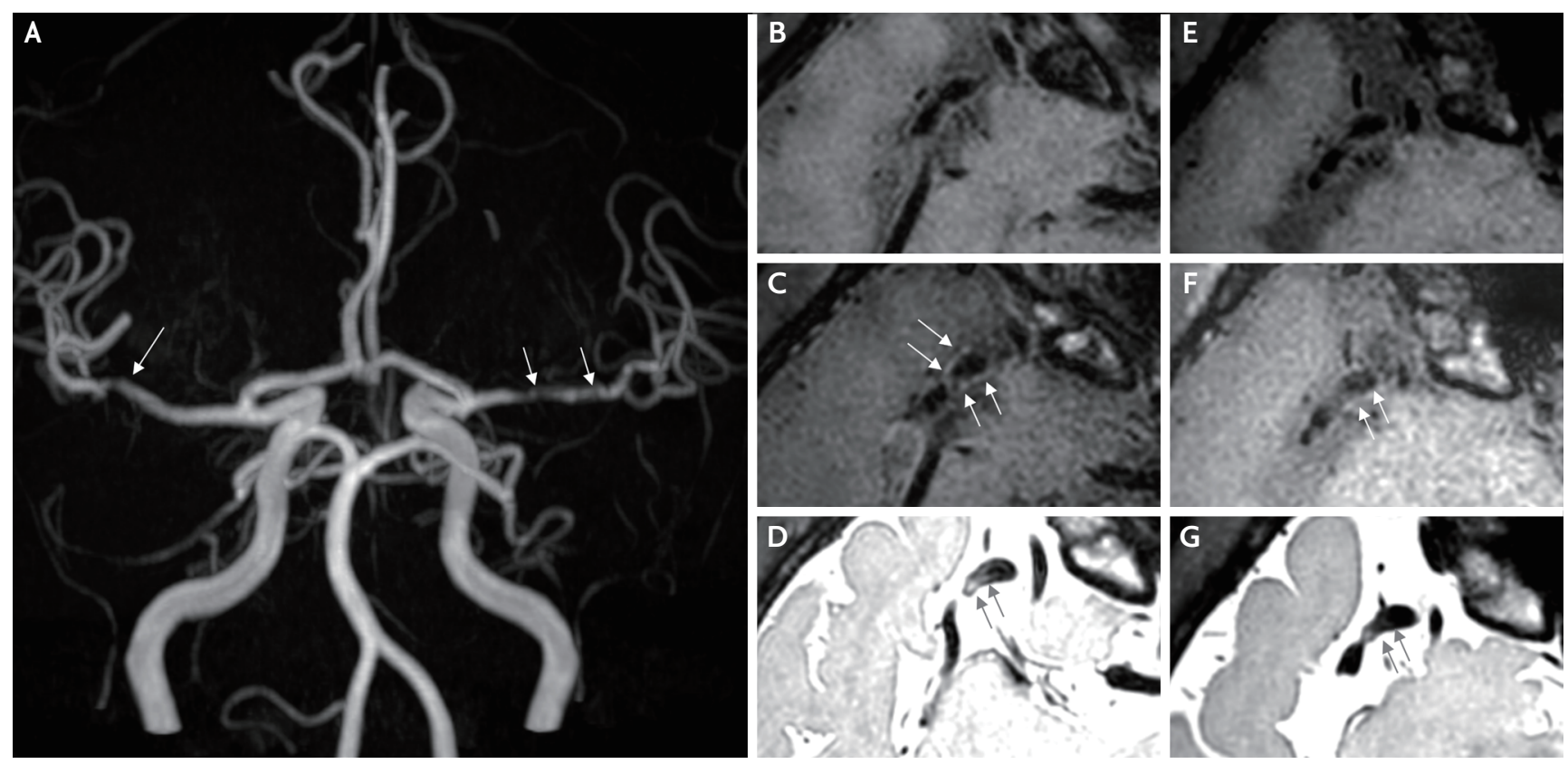

FIG. 1. Vessel wall imaging (VWI) of the intracranial arteries was performed. (A) Bilateral MCA stenosis observed in the maximum intensity projection image (white arrows). (B) Stenosis of the right MCA bifurcation is observed on Tr weighted image (T1WI) (axial). (C) Subtle eccentric vessel wall enhancement of the right MCA bifurcation is observed on contrast-enhanced TIWI (axial) (white arrows). (D) High signal intensity in the right MCA wall is observed in the black blood proton density image (axial view) (black arrows). (E) Stenosis of the right MCA bifurcation is observed in T1 weighted image of the follow-up VWI (axial view). (F) Interval-decreased eccentric vessel wall enhancement of the right MCA bifurcation is observed in contrast-enhanced TIWI of follow-up VWI (axial) (white arrows). (G) Interval decreased high signal intensity in the right MCA wall in the black blood proton density image of the follow-up VWI (axial) (black arrows). 
ed vascular remodeling with an active inflammatory process. This correlates with the pathophysiology of nilotinib-associated vascular toxicity and consequentially progressed nilotinib-associated atherosclerosis. Therefore, it can be seen through this case that even in the absence of preexisting vascular risk factors, intracranial atherosclerosis could progress through taking nilotinib.

VWI is a non-invasive diagnostic method for evaluating vascular beds. In the VWI of Moyamoya disease and central nervous system vasculitis, concentric vessel wall thickening with high signal intensity in PDI is typically observed. Moyamoya disease typically involves the distal internal carotid artery to the MCA. In contrast, this case showed eccentric wall thickening with high signal intensity in PDI localized in the distal Mr segment. Although the RNF 213 gene test was not conducted, the possibility of Moyamoya disease seemed unlikely. In VWI of intracranial arterial dissection, dissection flap or tapered stenosis might be observed, and the regression of stenosis could be confirmed in the follow-up VWI. In contrast, in our case, a follow-up VWI showed decreased wall enhancement and a minimal change in the stenosis degree of both MCAs.

he reported incidence of vascular toxicity in TKIs varies according to each drug regimen., ${ }^{9,10}$ Although there is a lack of head-to-head comparisons, the reported risk of vascular toxicity was higher in the 400 mg nilotinib twice daily regimen than in the $100 \mathrm{mg}$ dasatinib daily regimen. Therefore, the medication was changed from $400 \mathrm{mg}$ nilotinib twice daily to $100 \mathrm{mg}$ dasatinib daily in this context.

In addition, regardless of the association with TKI treatment, medical treatment, including antiplatelet therapy, is considered the first-line treatment for symptomatic intracranial arterial stenosis. Intracranial percutaneous angioplasty is only offered as an option when hemodynamic impairment persists under aggressive medical treatment, whilst a few cases have reportedly been successfully treated with stenting. ${ }^{3}$

To the best of our knowledge, this is the first report of serial VWI with nilotinib-induced cerebral arterial stenosis. In this case, bilateral MCA stenosis progressed, even without significant vascular risk factors. Follow-up VWI after switching from nilotinib to dasatinib showed an interval decrease in vessel wall enhance- ment. Clinicians should pay attention to the possibility of nilotinib-induced CVD, since nilotinib may accelerate pro-inflammatory and pro-atherosclerotic processes. Furthermore, prompt management, including switching or discontinuation of the culprit is critical.

\section{Ethics Statement}

This study was approved by the Institutional Review Board (IRB) of Seoul National University Hospital (IRB No. 2110-063-1261). The IRB waived the need for informed consent.

\section{Availability of Data and Material}

The authors confirm that the data supporting the findings of this study are available within the article.

\section{Acknowledgments}

None.

\section{Sources of Funding}

None.

\section{Conflicts of Interest}

No potential conflicts of interest relevant to this article was reported.

\section{REFERENCES}

1. Pasvolsky O, Leader A, Iakobishvili Z, Wasserstrum Y, Kornowski R, Raanani P. Tyrosine kinase inhibitor associated vascular toxicity in chronic myeloid leukemia. Cardiooncology. 2015;1:5.

2. Chen CJ, Sorace BJ, Shakeri A, Park MS, Southerland AM, Worrall BB, et al. Tyrosine kinase inhibitor induced rapidly progressive vasculopathy after intracranial stent placement. J Neurointerv Surg. 2018;10:e28.

3. Ozaki T, Nakamura H, Izutsu N, Masaie H, Ishikawa J, Kinoshita M. Intracranial stenting for nilotinib treatment-associated cerebrovascular stenosis in chronic myeloid leukemia. Interv Neuroradiol. 2017;23:527-530.

4. Kakadia B, Thakkar R, Sanborn E, Suero-Abreu GA, Jovin TG, Then R. Nilotinib-associated atherosclerosis presenting as multifocal intracranial stenosis and acute stroke. $J$ Stroke Cerebrovasc Dis. 2021;30:105883.

5. Sukegawa M, Wang X, Nishioka C, Pan B, Xu K, Ohkawara 
$\mathrm{H}$, et al. The BCR/ABL tyrosine kinase inhibitor, nilotinib, stimulates expression of IL-1 $\beta$ in vascular endothelium in association with downregulation of miR-3p. Leuk Res. 2017;58:83-90.

6. Ito Y, Miyamoto T, Chong Y, Maki T, Akashi K, Kamimura T. Nilotinib exacerbates diabetes mellitus by decreasing secretion of endogenous insulin. Int J Hematol. 2013;97:135138.

7. Rea D, Mirault T, Cluzeau T, Gautier JF, Guilhot F, Dombret $\mathrm{H}$, et al. Early onset hypercholesterolemia induced by the and-generation tyrosine kinase inhibitor nilotinib in patients with chronic phase-chronic myeloid leukemia. Haematologica. 2014;99:1197-1203.
8. Hadzijusufovic E, Albrecht-Schgoer K, Huber K, Hoermann G, Grebien F, Eisenwort G, et al. Nilotinib-induced vasculopathy: identification of vascular endothelial cells as a primary target site. Leukemia. 2017;31:2388-2397.

9. Manouchehri A, Kanu E, Mauro MJ, Aday AW, Lindner JR, Moslehi J. Tyrosine kinase inhibitors in leukemia and cardiovascular events: from mechanism to patient care. Arterioscler Thromb Vasc Biol. 2020;40:301-308.

10. Hochhaus A, Saglio G, Hughes TP, Larson RA, Kim DW, Issaragrisil $\mathrm{S}$, et al. Long-term benefits and risks of frontline nilotinib vs imatinib for chronic myeloid leukemia in chronic phase: 5-year update of the randomized ENESTnd trial. Leukemia. 2016;30:1044-1054. 